\title{
Retraction Note to: The Impact of Survivin on Prognosis and Clinicopathology of Glioma Patients: A Systematic Meta-Analysis
}

\author{
Shunzeng Lv ${ }^{1,2} \cdot$ Congxin Dai $^{1} \cdot$ Yuting Liu ${ }^{3} \cdot$ Ranran Shi $^{2} \cdot$ Zhenyu Tang ${ }^{2}$. \\ Mingzhi Han ${ }^{2} \cdot$ Ruixiang Bian ${ }^{2} \cdot$ Bowen Sun $^{1} \cdot$ Renzhi Wang ${ }^{1}$
}

Published online: 16 January 2017

(C) Springer Science+Business Media New York 2017

\section{Retraction Note to: Mol Neurobiol (2015) 51:1462-1467 DOI 10.1007/s12035-014-8823-5}

This article has been retracted at the request of the Editor-inChief and the Publisher. The article shows evidence of irregularities in authorship during the submission process. There is strong reason to believe that the peer review process was compromised and the authors have plagiarized parts from the following article:

Shunzeng Lv, Ekaterina Turlova, Shigang Zhao, Huihui Kang, Mingzhi Han, Hong-Shuo Sun, Prognostics and clinicopathological significance of survivin expression in bladder cancer patients: a meta-analysis. Tumor Biol. (2014) 35: 1565. doi:10.1007/s13277-013-1216-y; Received: 23 July 2013

The online version of the original article can be found at http://dx.doi. org/10.1007/s12035-014-8823-5.

Renzhi Wang

wrzpumch@163.com

1 Department of Neurosurgery, Chinese Academy of Medical Sciences \& Peking Union Medical College, Peking Union Medical College Hospital, Beijing 100730, China

2 Shandong University School of Medicine, Jinan, Shandong, China

3 Department of Obstetrics and Gynecology, Chinese Academy of Medical Sciences \& Peking Union Medical College, Peking Union Medical College Hospital, Beijing, China
In addition, the article shows similarities with the following article which was submitted within a close timeframe:

Xiangshan Yang, Shunzeng Lv, Yuting Liu, Daotang Li, Ranran Shi, Zhenyu Tang, Jianzhen Fan, Zhongfa Xu, The Clinical Utility of Matrix Metalloproteinase 9 in Evaluating Pathological Grade and Prognosis of Glioma Patients: A Meta-Analysis. Mol Neurobiol (2015) 52: 38. doi:10.1007/s12035-014-8850-2;

Received: 20 July 2014

The article "The impact of survivin on prognosis and clinicopathology of glioma patients: a systematic meta-analysis" was received on 26 June 2014.

As such the validity of the content of this article cannot be verified. 